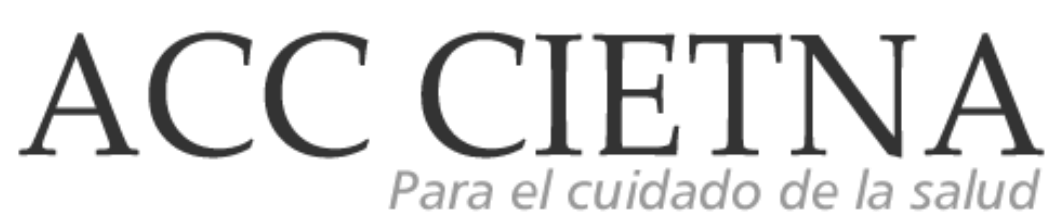

https://doi.org/10.35383/cietna.v2i2.142

\title{
Prevenção da Hepatite B em trabalhadores de saúde sob o olhar dos acadêmicos de Enfermagem Preventing hepatitis $B$ in health workers under the eye of nursing students
}

\section{Fonseca Martins Adriana ${ }^{1}$, Ortiz Sanchez Maritza Consuelo ${ }^{2}$, Ribeiro de Castro Magda ${ }^{3}$, Pereira Marilia de Jesus ${ }^{4}$}

\begin{tabular}{l} 
INFORMACIÓN DEL ARTÍCULO \\
\hline Historia del artículo: \\
Recibido el 23 de mayo de 2015 \\
Aceptado el 15 de noviembre de 2015
\end{tabular}

Palabras claves:

Enfermagem

Hepatite B

Saúde do Trabalhador.

\section{RESUMO}

O estudo objetivou analisar as ações do enfermeiro na prevenção da Hepatite B em trabalhadores de saúde. Pesquisa de abordagem qualitativa e descritiva, cujos entrevistados foram dez acadêmicos de Enfermagem. A análise temática revelou que, na visão dos colaboradores, a educação, com ênfase na utilização dos Equipamentos de Proteção Individual, e a vacinação são as principais ações dos enfermeiros na prevenção da Hepatite B. Estas atividades envolvem o reconhecimento e a participação dos trabalhadores, bem como o apoio institucional. Como conclusão a pesquisa reforça a relevância de maior conhecimento sobre a temática na formação dos profissionais de enfermagem, tendo como foco a promoção da saúde, a prevenção e a capacitação para a realização da vigilância epidemiológica para o controle da doença. Nesse sentido, as ações realizadas pelo enfermeiro visam contribuir diretamente na melhoria da qualidade de vida do trabalhador.

\footnotetext{
${ }^{1}$ Enfermeira do trabalho na empresa Tupperware. Rio de Janeiro, Brasil. Email:af@gmail.com

2 Doutora em Enfermagem, Professora Adjunta do Departamento de Enfermagem Fundamental da Escola de Enfermagem Aurora de Afonso Costa da Universidade Federal, Rio de Janeiro. Brasil. Email: mc@gmail.com

3 Doutoranda em Enfermagem. Professora Assistente da Universidade Federal de Espírito Santo de Brasil, Rio de Janeiro, Brasil. Emai: mribeiro@gmail.com

${ }^{4}$ Mestre em Enfermagem. Professora Assistente da Faculdade Cenecista de Rio das Ostras. Rio de Janeiro, Brasil. Email: mpereira@gmail.com
} 


\section{Preventing hepatitis $B$ in health workers under the eye of nursing} students

\section{ABSTRACT}

Keywords:

Nursing

Hepatitis B

Worker Health.
The study aimed to analyze the actions of the nurse in the prevention of Hepatitis B on health workers. The qualitative and descriptive search of approach, whose interviewed were ten Nursing students. Thematic analysis revealed that, in the view of collaborators, education, with emphasis on the use of Personal Protective Equipment and vaccination are the main actions of nurses in the prevention of Hepatitis B. These activities involve the recognition and worker participation as well as institutional support. As a conclusion the research strengthens the importance of better understanding on the subject in the training of nursing professionals focusing on health promotion, prevention and training that makes possible the realization of epidemiological surveillance for disease control. Accordingly, the actions taken by nurses aim to contribute directly in improving the quality of workers life.

\section{Introdução}

O presente trabalho tem por objeto de estudo a visão dos acadêmicos de Enfermagem de uma Instituição de Ensino Superior (IES) sobre as ações do enfermeiro na prevenção da Hepatite $B$ em trabalhadores de saúde. A motivação para realizar esta pesquisa se deve a relevância e reflexão da temática, após leituras de diversos estudos, nas quais os resultados evidenciam uma estimativa de que, nos países desenvolvidos, o risco de contrair Hepatite B é de 3 a 6 vezes maior entre trabalhadores da saúde quando comparado à população em geral, sendo que, nos países em desenvolvimento, o risco é de 6 a 18 vezes maior. Tal situação reflete o elevado índice de trabalhadores de saúde contaminados com esta doença que tem grande potencial de gerar complicações à saúde, contribuindo para que a cada ano, uma estimativa de 200 a 300 trabalhadores de saúde percam seus fígados 1,2 .
Nessa vertente, a Organização Mundial de Saúde (OMS) estima que cerca de 2 bilhões de pessoas no mundo já tiveram contato com o vírus da Hepatite B (HBV), e que 325 milhões tornaram-se portadores crônicos. Em termos mundiais, as taxas de prevalência da Hepatite B variam amplamente, de $0,1 \%$ a taxas superiores a $30 \%$, como as verificadas em países asiáticos 3 .

No entanto, é preciso considerar que muitos indivíduos infectados são assintomáticos e que as infecções sintomáticas são insuficientemente notificadas. Assim, a frequência da Hepatite B é, certamente, subestimada 3 .

Os indivíduos portadores do vírus estão expostos a complicações como a cirrose e o câncer de fígado, que podem ocasionar a necessidade de um transplante quando ocorre grande comprometimento hepático. Só no Brasil, dois milhões de pessoas sofrem da forma crônica de Hepatite B4. 
A Hepatite é uma doença grave, que afeta o fígado, um dos órgãos mais importantes do corpo humano, responsável pela transformação dos alimentos em energia, e atua como regulador do gasto da mesma segundo a necessidade do organismo. Se o fígado funciona bem, enfrentamos o cotidiano com muito mais disposição, se falha, ficamos cansados e sem disposição para realizar as atividades ${ }^{4}$. Entendido assim, a disfunção do fígado afeta todos os sistemas corporais, pelo que se faz necessário conhecer os diversos distúrbios e/ou as doenças que o afetam.

No que diz respeito à transmissão do vírus da Hepatite B (HBV), destaca-se que este é encontrado no sangue, saliva, sêmen e secreções vaginais, podendo ser transmitido principalmente através do sangue (vias percutânea e mucosa), bem como através de mães portadoras para seus filhos no momento do nascimento ${ }^{4}$.

Estudos mostram que o HBV é mais facilmente transmitido e apresenta maior grau de infectividade quando comparado ao vírus da Hepatite C (HCV) e ao vírus da SIDA, ao considerar sua resistência de sobrevivência de 7 dias em ambiente externo ${ }^{4,5}$.

Um elevado número de indivíduos (> 90\%) que contraem infecções por Hepatite B desenvolverão anticorpos e irão recuperar-se espontaneamente em seis meses. Outros $10 \%$ dos portadores desta infecção progridem para a Hepatite crônica, com infecção persistente por HBV e inflamação e lesão hepatocelular. Assim, a doença permanece como uma importante causa de cirrose e de carcinoma hepatocelular em nível mundial ${ }^{4}$.

No que concerne à transmissão ocupacional da Hepatite B, ressalta-se que após um acidente percutâneo, o risco é de $30 \% 2$. Os acidentes com agulhas e outros perfuro cortantes usados nas atividades laboratoriais e de assistência à saúde estão associados à transmissão ocupacional de mais de 20 diferentes patógenos, entre eles o vírus da Hepatite B (HBV), o vírus da Hepatite C (HCV) e o vírus da Síndrome da Imunodeficiência Adquirida $(\mathrm{SIDA})^{6}$.

Estudos sobre a Hepatite $B$ também demonstram que a prevalência deste vírus é maior entre os profissionais da saúde, como médicos e técnicos, pessoal de laboratório e trabalhadores de serviços de higienização/limpeza, quando comparados à população em geral. Nesse contexto, a equipe de enfermagem constitui um grupo ocupacional relevante ao tratar desse assunto, especialmente porque é o maior segmento da força de trabalho em muitos hospitais 2,6 .

$\mathrm{Na}$ área hospitalar, os acidentes com materiais perfuro cortantes são frequentes entre os profissionais de enfermagem, visto que são estes os que executam atividades diretas durante 24 horas ininterruptas e em contato direto com os pacientes. Apesar de os hospitais serem entidades que visam à assistência, o tratamento e a cura de pessoas acometidas por doenças também podem ser responsáveis pelo adoecimento daqueles que ali trabalham ${ }^{5}$.

Desse modo, faz-se necessário refletir acerca da prevenção de acidentes de trabalho como uma medida que pode, mais significativamente, diminuir a exposição ocupacional aos diversos riscos, entre eles, o biológico. A enfermagem pode orientar algumas medidas, tais como uso de dispositivos de segurança e vacinação; promover periodicamente treinamento em serviço; diminuir os riscos de contaminação, além de permitir que os trabalhadores consigam decodificar a organização de trabalho em que estão inseridos, encontrando, assim, soluções para sua prática diária6.

No Brasil, o Ministério da Saúde desenvolve o combate às Hepatites em três frentes: prevenção e controle, vigilância epidemiológica e assistência ao portador. Nesse contexto, o Programa Nacional de Hepatites Virais tem como objetivos desenvolver essas ações de prevenção, promover a vigilancia epidemiológica e sanitária, garantir o diagnóstico 
e o tratamento das hepatites, ampliar o acesso ao tratamento, melhorar a qualidade e a capacidade dos serviços de saúde e acompanhar e avaliar o conjunto das ações ${ }^{3}$.

O Programa para Prevenção e Controle das Hepatites Virais nasceu a partir de um movimento dos profissionais da área de saúde, de Organizações não Governamentais (ONGs) e da sociedade. As ações vêm sendo implantadas de forma heterogênea, conforme a realidade de cada estado ${ }^{3}$.

A prevenção da Hepatite B é feita com a aplicação de três doses da vacina, em que a primeira é administrada ao nascer, a segunda, ao final do primeiro mês de vida, e a terceira, aos seis meses. A vacina também é oferecida para pessoas na faixa etária de 1 a 19 anos, bem como para os grupos de risco, como os imunodeprimidos, os profissionais da área de Saúde e os profissionais do sexo, em qualquer faixa etária. Para os trabalhadores da saúde, o Ministério da Saúde recomenda que, 30 dias após a administração da última dose do esquema vacinal contra a Hepatite $B$, sejam realizados exames sorológicos para controle dos títulos de anticorpos ${ }^{3}$.

A triagem continuada de doadores de sangue para a presença de antígenos da Hepatite B diminuirá ainda mais o risco de transmissão por transfusão sanguínea. No laboratório clínico, as áreas de trabalho deverão ser desinfetadas diariamente, e a ingestão de alimentos e fumo é proibida neste ambiente e em outras áreas expostas ao material biológico 4

Existe uma necessidade de fazer intervenções quanto à prevenção da Hepatite $B$ no ambiente de trabalho pelo (a) enfermeiro (a), para diminuir o alto índice de contaminação. Nesse sentido é de suma importância para a equipe de enfermagem a vacinação e o conhecimento da imunidade à Hepatite $B^{5}$.
Rotinas de treinamento devem ser adotadas permanentemente como programas preventivos de saúde e de segurança de trabalho pelo (a) enfermeiro (a), visando aumentar o conhecimento quanto à tomada de decisões correta em caso de exposições do profissional de saúde a sangue e fluidos corporais. O mesmo deve orientar sua equipe antes de iniciar qualquer atividade profissional, esclarecendo e reforçando sempre as necessidades à prevenção de acidentes no âmbito de trabalho através do uso de equipamentos de proteção coletiva (EPC) e equipamentos de proteção individual (EPI), que constitui uma barreira protetora para o trabalhador. A educação em relação ao emprego correto de proteção individual e quanto à vacinação adequada devem ser realizadas pelo enfermeiro, previamente ao ingresso do profissional de saúde em sua prática diária 5 .

Frente ao exposto, esta pesquisa tem por objetivo analisar as ações do enfermeiro na prevenção da Hepatite B em trabalhadores de saúde.

Este estudo visa preencher as lacunas existentes relacionadas a temática aqui investigada, com vistas a contribuir para reflexão e conhecimento sobre as ações do profissional da enfermagem, especialmente no que tange a promoção, prevenção e controle da contaminação por Hepatite B entre os profissionais de saúde.

\section{Método}

Pesquisa descritiva, dotada de abordagem qualitativa, considerada como um mundo de significados passível de investigação em que a linguagem dos autores sociais e suas práticas são as matérias-primas da abordagem. São os níveis de significados,motivos, aspirações, atitudes, crenças e valores que se expressam pela linguagem comum e na vida cotidiana, tendo a possibilidade de conhecimento gerado a partir de pesquisa de campo ${ }^{7}$. O presente estudo nos possibilitou descrever as ações que o enfermeiro 
realiza na prevenção da Hepatite $B$, tais como são definidas e ou entendidas pelos próprios sujeitos ${ }^{8}$.

Os colaboradores do estudo foram 10 acadêmicos de Enfermagem do oitavo período de uma instituição de ensino superior da Cidade do Rio de Janeiro. Utilizaram- se como critérios de inclusão os discentes matriculados na disciplina de Estágio Supervisionado, uma vez que esta possibilita colocar em prática os conhecimentos e habilidades adquiridas durante a formação acadêmica.

A coleta dos dados foi realizada através de entrevista semiestruturada, mediante a utilização de um roteiro elaborado acerca do objetivo da pesquisa. Com a entrevista emergiram informações de forma mais livre e as respostas não estiveram condicionadas a uma padronização de alternativas. ${ }^{9}$

As entrevistas foram gravadas e depois se procedeu à ordenação dos dados, o que exigiu, em um primeiro momento, a transcrição, seguida da leitura e releitura do material, tornando viável a organização dos achados?.

A pesquisa atendeu as exigências científicas e éticas acatando ao preconizado acerca da ética em pesquisa com seres humanos, na qual os colaboradores assinaram o Termo de Consentimento Livre e Esclarecido, segundo a Resolução 466/2012.

Para análise dos dados aplicou-se a análise temática, que visa descobrir os núcleos de sentido que compõem uma comunicação, cuja presença ou frequência signifiquem alguma coisa para o objeto analítico visado, ou seja, qualitativamente, a presença de determinados temas denotam os valores de referência e os modelos de comportamento presentes no discurso ${ }^{7}$.

\section{Resultados, análise e discussão}

Dos discursos dos colaboradores emergiu a seguinte categoria temática: Ações do enfermeiro na prevenção da Hepatite $B$ em trabalhadores de saúde, e duas subcategorias: Educação e Vacinação.

Ações do enfermeiro na prevenção da Hepatite B em trabalhadores de saúde:

Educação

É incontestável o papel do enfermeiro como educador em saúde, seja em sua atuação individual ou coletiva. Como educador ele tem em vista o objetivo de conscientização dos trabalhadores e visa proporcionar-lhes a oportunidade ativa no processo de ensinoaprendizagem, de modo a se tornarem agentes de mudanças. O enfermeiro educador trabalha no processo que envolve a interação entre educadores e educandos, e não uma simples transmissão de conhecimentos, um discurso, de alguém mais "competente" para outro "menos competente". A educação "é uma via de mão dupla" em que educadores e educandos constroem um conhecimento em comum, através de uma reflexão crítica ${ }^{10}$.

Este entendimento foi mostrado pela maioria dos colaboradores, que referem que o enfermeiro na prevenção da Hepatite B deve atuar como educador:

[...] tem o papel de educador, promovendo ações necessárias para os trabalhadores de saúde [...] (Depoente 1)

[...] eu vejo muito o enfermeiro como educador [...] educar para a saúde [...] (Depoente 4) O enfermeiro tem a função de educador, ele tem que interferir para que o profissional de saúde tenha noção da importância de se proteger, de cuidar de si mesmo [...] (Depoente 5)

[...] o papel do enfermeiro então seria com palestras, educação permanente, porque não adianta somente dar a palestra é preciso persistir 
para que as pessoas tomem consciência [...] (Depoente 6)

[...] o papel do enfermeiro é de educador [...] então eu acho importantíssimo a divulgação através de palestras. (Depoente 9)

O maior papel do enfermeiro nos outros colegas que trabalham na mesma área é educar buscando mudanças de comportamentos [...] (Depoente 10)

A educação para o cuidado em saúde, tanto dos pacientes, quanto dos estudantes e da equipe, é considerada um processo político pedagógico, requerendo o desenvolvimento de um pensar crítico e reflexivo, permitindo desvelar a realidade e propor ações transformadoras que levem o indivíduo a sua autonomia e emancipação enquanto sujeito histórico e social, capaz de propor e opinar nas decisões de saúde para cuidar de si mesmo, de sua família e da coletividade ${ }^{11}$.

É essencial que o profissional enfermeiro esteja efetivamente preparado para prestar serviços educativos que atendam as necessidades de muitos indivíduos e grupos em diferentes circunstâncias, nos diversos campos de prática.

O exercício de uma prática educativa crítica, como experiência especificamente humana, constitui uma forma de intervenção no mundo comprometida com o princípio de democracia que rejeita qualquer forma de discriminação, dominação e integra uma atitude de inovação e renovação na crença de que é possível mudar. Assim, para educar em saúde, se faz necessário estar aberto ao contorno geográfico, social, político, cultural do indivíduo, família e comunidade ${ }^{10}$.

O conceito de educação em saúde está ancorado no conceito de promoção da saúde, que trata de processos que abrangem a participação de toda a população no contexto de sua vida cotidiana e não apenas das pessoas com risco de adoecer ${ }^{11}$

A educação em saúde destaca-se como estratégia de promoção à saúde neste processo de conscientização individual e coletiva de responsabilidades e de direitos à saúde, estimulando ações que atendam aos

\section{Considerações finais}

princípios do Sistema Único de Saúde (SUS). A partir deste enfoque, é necessário eleger estratégias didáticas que conduzam a uma transformação dos indivíduos socialmente inseridos no mundo, ampliando sua capacidade de compreensão da complexidade dos determinantes de ser saudável ${ }^{10}$.

Uma educação em saúde nos moldes da integralidade inclui políticas públicas, ambientes apropriados para além dos tratamentos clínicos e curativos, comprometidas com o desenvolvimento da solidariedade e da cidadania, envolvidas na melhoria da qualidade de vida e na promoção do homem. A partir do princípio de integralidade, concebe-se que todos os profissionais que trabalham com o ser humano devem aprimorar uma visão holística- ecológica' ${ }^{1}$.

Importante ressaltar que o sucesso de qualquer programa educativo está diretamente ligado à participação e reconhecimento por parte dos trabalhadores e apoio da instituição onde o mesmo desenvolve suas atividades ${ }^{12}$.

Ainda no que concerne o papel do enfermeiro como educador, alguns depoentes referem que para prevenir a Hepatite B fazem-se necessárias orientações quanto à utilização dos Equipamentos de Proteção Individual (EPIs), conforme os relatos a seguir:

\section{[...] importante orientar a correta utilização do EPI, descarpack [...] (Depoente 1)}

\section{[...] importante educar para que os trabalhadores utilizem EPI (Depoente 2)}

[...] importância de se proteger usando o EPI [...]

(Depoente 5)

\section{[...] orientações para utilização de EPI (Depoente 7)}




\section{[...] orientar que tem que usar EPI [...] (Depoente} 10)

Destaca-se que os Equipamentos de Proteção Individual (EPI) são todos os dispositivos de uso individual, de fabricação nacional ou estrangeira, destinados a proteger a saúde e a integridade física do trabalhador, devendo ser fornecidos gratuitamente pela empresa para todos os trabalhadores, sempre que as medidas de proteção coletiva forem inviáveis ${ }^{13}$.

Contudo, tem-se como agravante a hipótese de que a prevenção e controle dos agentes biológicos ainda são negligenciados pela maioria dos trabalhadores por desconhecerem os riscos inerentes a tais agentes; a falta de educação continuada através de treinamento em serviço; a resistência dos profissionais em aderir o uso correto do EPI; os esforços e as precárias condições de trabalho a que se submetem os trabalhadores de saúde, como fatores que contribuem para ocorrência de acidentes por cargas biológicas ${ }^{14,15}$.

Nesse sentido, é necessária a intervenção com educação continuada a fim de diminuir e evitar os acidentes, sendo necessário esclarecer os profissionais sobre os métodos de prevenção adequados, tais como o uso dos EPI (óculos, máscara, capote, luvas, sapatos fechados, outros), bem como a lavagem das mãos e vacinação. $O$ uso de EPI minimiza a exposição ao risco, mas não elimina a possibilidade de contaminação diante de um acidente com perfuro cortante ${ }^{15}$.

Importante ressaltar que uma das depoentes considerou que, além de orientar quanto à utilização dos EPIs, os trabalhadores devem ser orientados quanto a não encapar agulha após utilização, com a finalidade de diminuir a exposição dos trabalhadores a agentes patógenos causados por acidentes com material perfuro cortante.

\section{[...] como não encapar agulha após utilização [...] (Depoente 5).}

No que diz respeito ao manuseio de agulhas, os profissionais devem seguir algunas recomendações: as agulhas não devem ser entortadas, quebradas, recapeadas ou removidas da seringa após o uso, para se evitar a autoinoculação e produção de aerossóis. Agulhas utilizadas devem ser imediatamente colocadas em recipientes resistentes a perfurações e autoclavados antes do descarte ${ }^{15}$.

No Brasil, existe uma Norma Regulamentadora que tratado EPI, condicionando seu uso e comercialização à obtenção de Certificado de Aprovação (CA) expedido pelo Ministério do Trabalho e Emprego, que define os procedimentos para o fabricante interessado em comercializar EPI. Esta norma ainda determina como competência do empregador: adquirir o EPI adequado ao risco de cada atividade; exigir seu uso; fornecer ao trabalhador somente o EPI aprovado pelo órgão nacional competente em matéria de segurança e saúde no trabalho; orientar e treinar o trabalhador sobre o uso adequado, a guarda e a conservação; substituir imediatamente quando danificado ou extraviado; responsabilizar-se pela higienização e manutenção periódica e comunicar ao Ministério do Trabalho e Emprego (MTE) qualquer irregularidade observada ${ }^{13}$.

Por outro lado, a norma regulamentadora em questão também aborda as responsabilidades do empregado quanto ao EPI, com destaque para ser utilizado apenas para a finalidade a que se destina; responsabilizar-se pela guarda e conservação; comunicar ao empregador qualquer alteração que o torne impróprio para uso e cumprir as determinações do empregador sobre o uso adequado $^{13}$.

\section{Vacinação}

Importante destacar que a vacina é considerada uma medida prioritária de proteção individual, e, no caso da Hepatite B confere imunidade em $90 \%$ dos vacinados ${ }^{12,15}$. 
Conforme discursos dos colaboradores, a vacinação da Hepatite B constitui uma das ações da enfermagem para a prevenção desta doença, conforme os relatos elucidam:

Em minha opinião as ações de enfermagem [...] é a vacinação contra a Hepatite B[...] (Depoente 3 )

[...] passar para os profissionais sobre a importância da vacina, porque deve se vacinar. (Depoente 4)

\section{[...] para passar para os trabalhadores o modo de prevenção e fazendo a vacinação (Depoente 8)}

O Programa Nacional de Imunizações (PNI), na área da saúde, é uma prioridade nacional, com responsabilidades dos governos federal, estadual e municipal. $\mathrm{O}$ alcance dos objetivos e a adoção de estratégias com um mínimo de unidade exigem a articulação dessas instâncias, de forma a compatibilizar atividades, necessidades e realidades, num esforço conjunto ${ }^{17}$.

A redução da morbidade e da mortalidade por imunização só será possível se os índices de cobertura forem altos e homogêneos. Para isto, é de suma importância que as equipes de saúde e, mais especificamente as equipes de vacinação, trabalhem com base em conhecimentos e práticas que tornem a sua ação mais eficaz e eficiente possível ${ }^{10}$.

Um melhor desempenho dos serviços de saúde é, certamente, resultado de um bom planejamento e de uma adequada infraestrutura de serviços. Em vacinação, particularmente, isso se traduz, por exemplo: numa rede de frio sem problema de manutenção; em pessoal treinado; em boa organização do trabalho; em registros confiáveis; em disponibilidade de vacinas ${ }^{17}$.

A vacinação é uma ação preventiva oferecida à população e sua utilização por essa mesma população depende, necessariamente, de decisão pessoal em ir se vacinar. O serviço de saúde não pode sozinho, interferir ou influenciar no poder de decisão, que é pessoal e intransferível, com isso, as equipes de saúde precisam contar com o apoio e a participação da população ${ }^{17}$.

Neste caso, os próprios trabalhadores de saúde precisam também contribuir conscientizando-se de que saúde é um direito, um direito que inclui a vacinação. Para isso, é necessário que estes profissionais adotem uma atitude mais ativa em busca da vacinação, considerando a atenção à saúde de forma global, pois torna-se difícil tratar apenas de uma questão, quando ter saúde é algo bem mais complexo ${ }^{15}, 17$.

No caso da Hepatite B, após completar o esquema, recomenda-se o teste sorológico - anti-HBs, para verificar a resposta vacinal. Essa conduta pode ser verificada no depoimento a seguir:

[...] além da vacina, deve-se fazer teste sorológico depois [...] não consigo criar anticorpos contra Hepatite B [...] (Depoente 6)

O teste sorológico deve ser realizado de um a dois meses após a última dose da vacina, devendo ser recomendado para os trabalhadores que se encontram em situação permanente de exposição ao sangue ${ }^{15}$.

Embora recomendado, este teste não está disponível gratuitamente na rede de saúde pública para os profissionais ${ }^{15}$.

Faz-se necessário acrescentar nessa discussão a importância do combate a Hepatite B, conforme o Programa Nacional de Hepatites Virais, a mesma das di ret ri zes a serem desenvol vi das na prevenção, vigilância epidemiológica e sanitária, entre outras.

A vacinação contra a Hepatite $B$, bem como a melhoria das condições de higiene e de saneamento das populações estão entre os avanços importantes. Para isso, as condições referentes à heterogeneidade socioeconômica, a distribuição irregular dos serviços de saúde, e a incorporação desigual de tecnologia avançada para diagnóstico e tratamento de enfermidades, 
são elementos importantes que devem ser considerados na avaliação do processo endemoepidêmico das hepatites virais ${ }^{17}$.

A identificação de pessoas com infecção crônica pelo HBV através de um diagnóstico precoce pode reducir a transmissão continuada da infecção, e o tratamento antiviral diminui o risco de evolução para cirrose e para o carcinoma hepatocelular ${ }^{17}$.

Para a diminuição de contaminação da Hepatite B, é importante elaborar e implementar um programa de educação continuada que aborde a questão dos acidentes e exposição à material biológico, esclarecendo os trabalhadores sobre a importância da notificação, busca de atendimento médico nas 2 horas que seguem o acidente, sensibilização sobre a eficácia da vacina para prevenção da Hepatite B, esclarecimento sobre a utilização de EPI e precauções padrão ${ }^{15}$.

O Ministério da Saúde criou, em 2002, o Programa Nacional para a Prevenção e o Controle das Hepatites Virais (PNHV), que contribui para o aprimoramento do conjunto de ações de saúde relacionadas às hepatites. A grande importância das hepatites não se limita ao enorme número de pessoas infectadas; estende-se também às complicações das formas agudas e crônicas ${ }^{17}$.

A vigilância epidemiológica das hepatites em nosso país utiliza o sistema universal e passivo, baseado na notificação compulsória dos casos suspeitos. Embora o sistema de notificação tenha apresentado melhoras, ele ainda é insatisfatório.

As principais questões a serem investigadas, e que podem contribuir para o melhor controle das hepatites, estão relacionadas à definição dos diferentes tipos de vírus e das doenças que determinam. O conhecimento adequado sobre a frequência do vírus $B$ e a implementação de estratégias indicadas para a sua prevenção exigem métodos complexos de vigilância epidemiológica.

Através da monitorização dos indivíduos recentement e infectados podem- se obter informações críticas para identificar com segurança surtos de Hepatite B. Embora pouco frequentes, há descrição de surtos em hospitais, envolvendo transmissão de pacientes a pacientes, através de equipamentos contaminados, frascos de medicamentos multiuso e transplantes de órgãos, entre outros 18 .

\section{Considerações finais}

Evidenciou-se neste estudo que os colaboradores identificaram as diversas ações que o enfermeiro deve realizar visando à prevenção da Hepatite $B$ em trabalhadores da saúde, com atenção prioritária para educação e vacinação.

A educação foi considerada como uma das ações principais, estando diretamente ligada à participação e ao reconhecimento dos trabalhadores, bem como o apoio da instituição. Ainda no que diz respeito à educação, houve destaque para a utilização de EPI, os quais têm a finalidade de diminuir a exposição, prevenir a contaminação e evitar os acidentes.

No decorrer da análise dos dados identificou-se a vacinação como outra das ações que o enfermeiro realiza; procedimento que visa à redução da morbidade e da mortalidade pela Hepatite B.

Entretanto, é importante que o trabalhador considere também a necessidade imperiosa de se proteger.

Este estudo reforça que é de extrema importância, na formação dos profissionais de enfermagem, obter maior conhecimento sobre as ações que este profissional realiza com vistas à promoção da saúde, prevenção e controle da Hepatite B. Os enfermeiros devem estar capacitados para realizar a vigilância epidemiológica como uma forma de controle da doença, visto que a grande importância das hepatites não se limita a alta incidência de casos, mas também às complicações na saúde do indivíduo portador do vírus.

Entende-se assim que as ações realizadas pelo enfermeiro na prevenção da Hepatite B são 
primordiais, já que estas contribuem diretamente na melhoria da qualidade de vida destes profissionais.

\section{Referências bibliográficas}

1. Paulino DCR, Lopes MVO, Rolim ILTP. Biossegurança e acidentes de trabalho com perfuro cortantes entre os profissionais de enfermagem de Hospital Universitário de Fortaleza - CE. Cogitare Enferm 2008 Out $\backslash$ Dez; 13(14): 507-13.

2. Bulhões I. Riscos do trabalho de enfermagem. Rio de Janeiro: SPC, 1994.

3. Ministério da Saúde. Secretaria de Vigilância em Saúde. Guia de Vigilância Epidemiológica.7ed. Brasília: Ministério da Saúde, 2009

4. Smeltzer SC, Bare BG. Brunner \& Suddarth, Tratado de enfermagem médico-cirúrgico. 12. ed. v.2. Rio de Janeiro: Guanabara Koogan; 2012.

5. Pinheiro J, Zeitoune RCG. Hepatite B: Conhecimento e medidas de biossegurança e a saúde do trabalhador de enfermagem. Esc Anna Nery Rev Enferm 2008 Jun; 12(2): 258-64.

6. Rapparine C, Érica RL. Programa de prevenção de acidentes com materiais perfuro cortantes em serviços de saúde. São Paulo: Funda Centro; 2008.

7. Minayo, Maria Cecília de Souza. O desafio do conhecimento: pesquisa qualitativa em saúde. 11 . ed. São Paulo: HUCITEC-ABRASCO; 2008.

8. Polit D, Hungler B. Fundamentos de pesquisa em enfermagem: métodos, avaliação e utilização. 7. ed. Porto Alegre: Artmed; 2011.

9. Manzini EJ. Uso da entrevista em dissertações e teses produzidas em um programa de pósgraduação em educação. Revista Percurso-NEMO. Maringá, 2012 v. 4,n.2, p.149-17.
10. Magalhães CR, Guimarães EC, Aguiar BGC. O papel do enfermeiro educador: ação educativa do enfermeiro no pré e pós-operatório. Revista de pesquisa: cuidado é fundamental, 2008; 115-19.

11. Machado MFAS, Monteiro EMLM, Queiroz DT, Vieira NFC, Barroso MGT. Integralidade, formação de saúde, educação em saúde e as propostas do SUS: uma revisão conceitual. Ciência e Saúde Coletiva 2007; 12(2): 335-42.

12. Silva, GMda; Seiffert, OMLB. Educação continuada em enfermagem: uma proposta metodológica. Rev. bras. enferm. vol.62 no.3 Brasília May/June 2009.

13. BRASI L. Mi ni s t ér i o do Tr abal ho. NR6. Equipamentos de proteção individual - EPI. 2001.

14. Garcia, GFB (org) Legislação de Segurança e a medicina do trabalho. 2 ed. São Paulo: Método. 2008

15. Ministério da Saúde. Manual de procedimento para vacinação. Disponível em: $<$ http://portal.saude.gov.br/portal/arquivos/pdf/ manu_proced_vac.pdf $>$. Acesso em 05 out. 2013.

16. Veiga MM, Duarte FJCM, Meirelles LA, Garrigou A, Baldi I. A contaminação por agrotóxicos e os equipamentos de proteção individual. Rev. bras. Saúde ocup. 2007 abri \agos; 32(116): 57-68.

17. Souza ACS, Alves SB, Santos SLV, Tipple AFV, Neves HCC, Barreto RASS. Adesão a vacina contra hepatite $B$ entre recém-formados da área de saúde do município de Goiânia. Cienc Cuid Saúde 2008 Jul $\backslash$ Set;7(3):363-69.

18. Marziale, M. H. P., Rodrigues, C. M. A produção científica sobre os acidentes de trabalho com material perfuro cortante entre trabalhadores de enfermagem. Rev Latino-am Enfermagem, jul-ago, 2002. 